\section{General principles of carotid Doppler ultrasonography}

Whal Lee

Department of Radiology, Seoul National University College of Medicine, Seoul, Korea

Carotid Doppler ultrasonography is a popular tool for evaluating atherosclerosis of the carotid artery. Its two-dimensional gray scale can be used for measuring the intima-media thickness, which is very good biomarker for atherosclerosis and can aid in plaque characterization. The plaque morphology is related to the risk of stroke. The ulceration of plaque is also known as one of the strong predictors of future embolic event risk. Color Doppler ultrasonography and pulse Doppler ultrasonography have been used for detecting carotid artery stenosis. Doppler ultrasonography has unique physical properties. The operator should be familiar with the physics and other parameters of Doppler ultrasonography to perform optimal Doppler ultrasonography studies.

Keywords: Carotid arteries; Atherosclerosis; Ultrasonography; Ultrasonography, Doppler; Plaque, atherosclerotic

\section{Starting Carotid Ultrasonography: Patient Position}

For carotid ultrasonography, there are two options for the relative position between the patient and examiner. One is the overhead position, in which the examiner sits beyond the patient's head beside the end of the examination table and use two hands for ultrasonography. In this position, the examiner should use his right hand for the right carotid artery and use his left hand for the left carotid artery. The benefit of this position is that the examiner can use both hands and there are plenty of positions possible for the ultrasonography probe. The sonic window can be made wider and offers a clear view of the carotid artery especially from the posterolateral projection. The examiner should be familiar with using both hands, which requires some practice.

Another position is the usual lateral sitting position, which is used for most other ultrasonography examinations. The examiner uses his right hand for both carotid arteries. This position makes it easy to control the machines. However, the right posterior projection is a bit more difficult. Between these two choices, the overhead position for Doppler ultrasonography of the carotid artery is recommended.

A pillow is not necessary. In fact, it produces a poorer window for the carotid artery. The optimal patient head position is tilted about $45^{\circ}$ away from the artery being examined. The neck of the patients should be relaxed. Contractions of the sternocleidomastoid muscle cause poor sonic penetration and make positioning of the probes difficult.

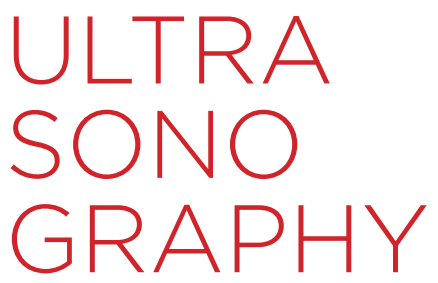

REVIEW ARTICLE

http://dx.doi.org/10.14366/usg. 13018 pISSN: 2288-5919 • elSSN: 2288-5943

Ultrasonography 2014;33:11-17

Received November 18, 2013

Revised November 20, 2013

Accepted December 11, 2013

Correspondence to:

Whal Lee, MD, Department of Radiology, Seoul National University College of Medicine, 101 Daehak-ro, Jongno-gu, Seoul 110-744, Korea Tel. $+82-2-2072-2584$

Fax. $+82-2-743-6385$

E-mail:whal.lee@gmail.com

This is an Open Access article distributed under the terms of the Creative Commons Attribution NonCommercial License (http://creativecommons.org/ licenses/by-nc/3.0/) which permits unrestricted noncommercial use, distribution, and reproduction in any medium, provided the original work is properly cited.

Copyright (C) 2014 Korean Society of Ultrasound in Medicine (KSUM)

How to cite this article:

Lee W. General principles of carotid Doppler ultrasonography. Ultrasonography. 2014 Jan;33(1):11-17. 


\section{Carotid Artery Anatomy and Tips for Differentiating the Internal Carotid Arteries from the External Carotid Arteries}

The right carotid artery arises from the right brachiocephalic artery. Ultrasonography can show the most proximal segment of the common carotid artery. The left common carotid artery arises from the aortic arch. Ultrasonography cannot show the proximal segment of the left common carotid artery. The examiner should be able to differentiate the internal carotid artery (ICA) from the external carotid artery (ECA). The ICA is located posterior and lateral to the ECA. The ICA is slightly larger than the ECA. The ECA has branches such as the lingual artery, but the ICA does not. The Doppler spectrums from the ICA show a lower resistive pattern (Fig. 1A). The velocity difference between the systolic phase and diastolic phase of the ICA is smaller than that of the ECA. Another way to differentiate the ECA from the ICA is that during the acquisition of the ECA Doppler spectrum, placing the fingertips on the ipsilateral temporal artery generates a serration-like artifact on the Doppler spectrum from the ECA. This temporal artery tapping-induced artifact is not seen from the ICA. This so-called "temporal tapping" is a useful tool in differentiating the ICA from the ECA (Fig. 1B). Being certain of which is the ECA and the ICA is important in case one of them is occluded.

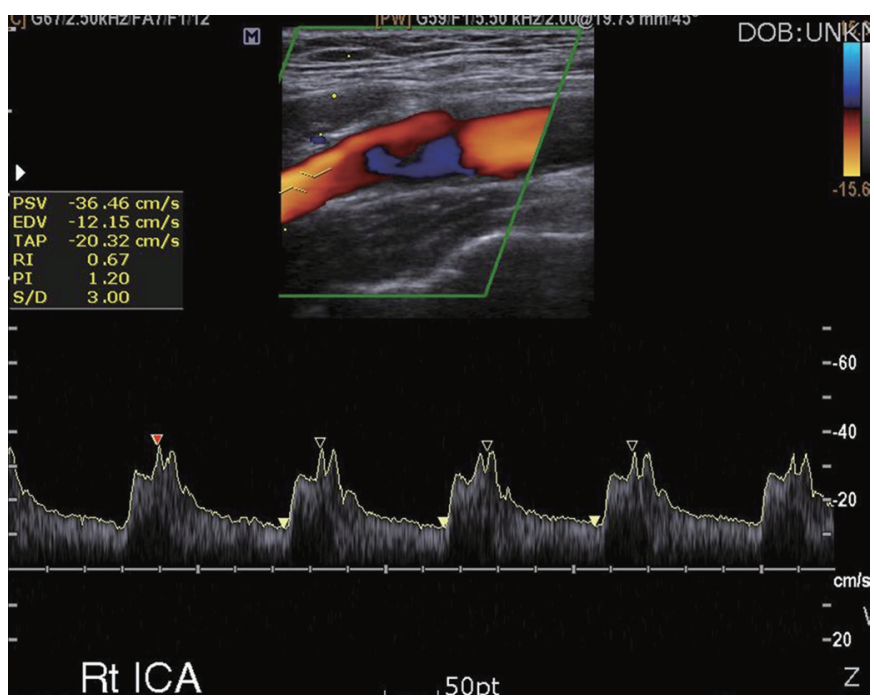

A

Fig. 1. Typical Doppler spectrum of the internal carotid artery and the external carotid artery.

A. The Doppler spectrum of the internal carotid artery shows a low resistance pattern with sufficient diastolic antegrade flow. B. The external carotid artery shows a more resistive pattern than the internal carotid artery. Another differentiating sign is temporal artery tapping. The Doppler spectrum of the external carotid artery without temporal tapping (S) and with temporal tapping (W) shows a difference in the wave form. The pressure of the temporal tapping is shown on the diastolic pulse spectrum of the external carotid artery. 


\section{Plaque Morphology and Plaque Volume}

The plaque morphology, such as the echogenicity of the plaque, the surface, presence of ulceration, as well as the presence of plaque and stenosis, is important for predicting future cardiovascular events [4]. A description of the plaque morphology from a gray-scale image is highly recommended during carotid Doppler ultrasonography (Fig. 3). The description should include the echogenicity of the plaque, the surface, and the presence of ulceration. The echogenicity of the plaque could be described as one of echogenic plaque, isoechoic plaque, echolucent plaque, or heterogeneous plaque. Isoechoic plaque means that the echogenicity of the plaque is the

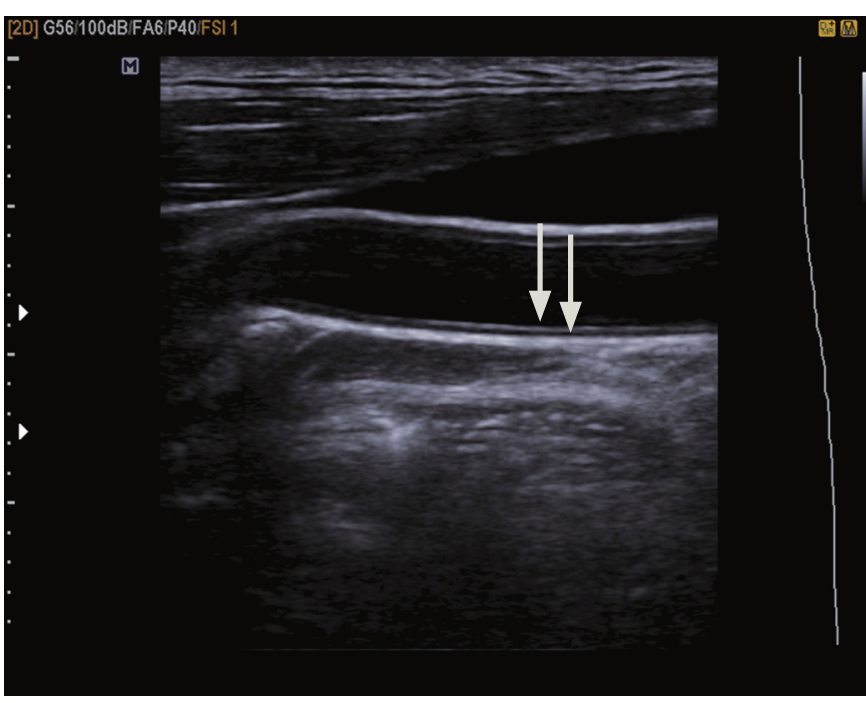

A

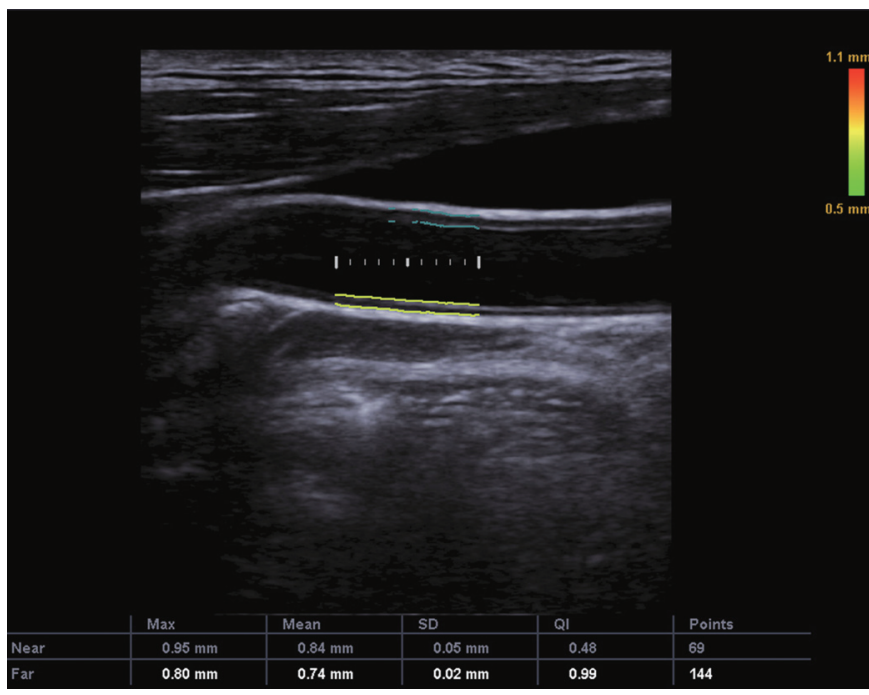

B

Fig. 2. Measuring of the intima-media thickness.

A. The distal carotid artery is the proper site for measuring the intima-media thickness. The two clearly visible hyperintense lines (arrows) that represent the interface between the blood pool and intima layer and the interface between the media layer and adventitial layer should be noted on the image. B. An example of automatic intima-media thickness measurement is shown.

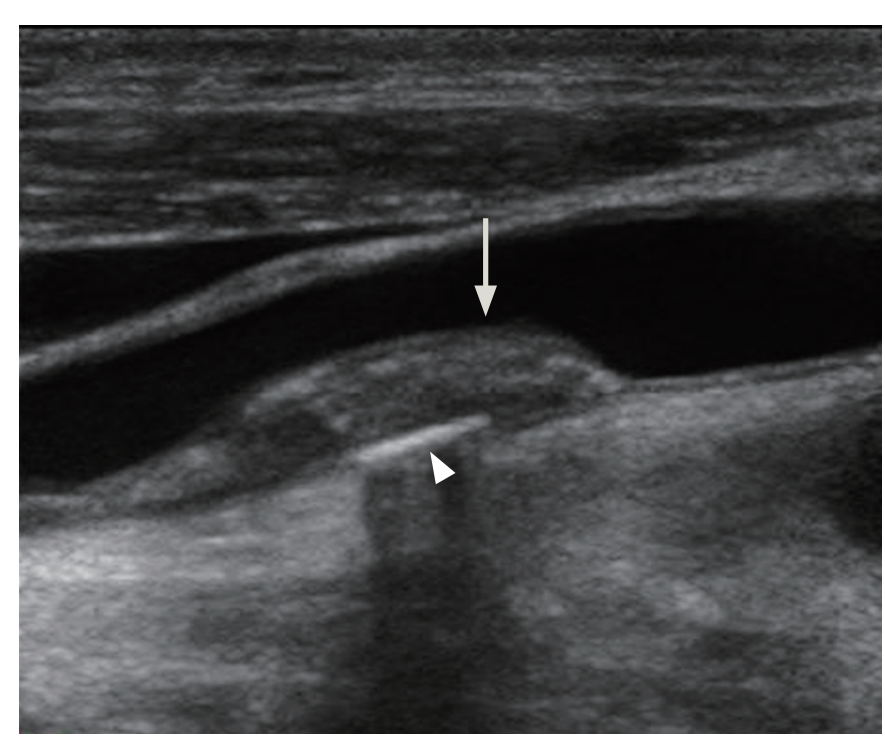

A

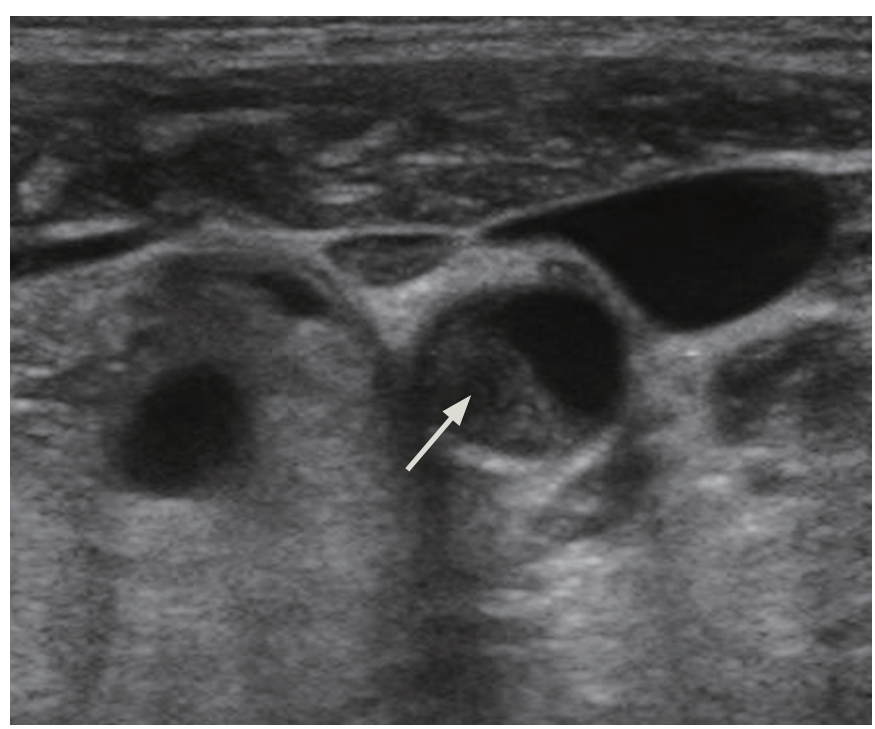

B

Fig. 3. The plaque morphology.

A. A gray-scale image of a longitudinal scan of the distal common carotid artery shows plaque with mixed echogenicity (arrow). A calcification is visible (arrowhead). The plaque surface is smooth. B. A transverse scan of the plaque at the distal common carotid artery shows central low echogenicity (arrow). The more lucent plaque is known to be associated with a higher risk of the stroke. 
same as that of the intima-media complex. The plaque surface can be described as smooth, irregular, or ulcerated. Plaque ulceration is associated with an increased risk of stroke $[5,6]$.

It is, however, very difficult to detect plaque ulceration by ultrasonography examination, and it is operator dependent. It is known that the sensitivity of detecting carotid plaque ulceration ranges from under $30 \%$ to over $80 \%$ when it is compared with pathological specimens $[7,8]$. The effort to detect ulceration should be applied to increase accuracy in the assessment of risk of the patient with carotid plaque. Another problem is that such an ulcer is not clearly correlated with symptoms and is difficult to detect

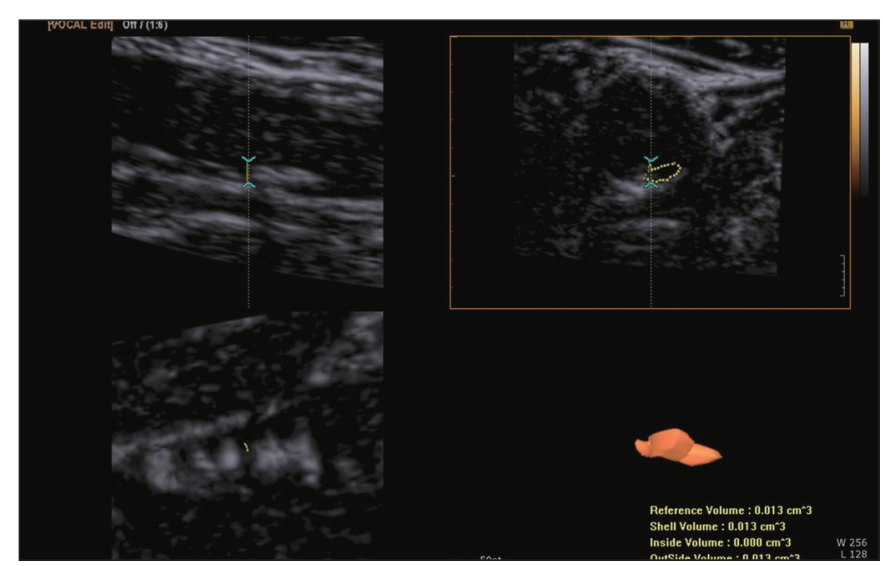

Fig. 4. Three-dimensional ultrasonography to measure the volume of the plaque. The total plaque volume can be measured by threedimensional ultrasonography. The plaque contour is traced manually and then the volume of the plaque is calculated. without careful gray-scale examination. The depression of the plaque surface by more than $2 \mathrm{~mm}$ is thought to indicate ulceration. The pattern of plaque ulceration can be cystic, bridge-shaped, spongeshaped, or a simple depression [8-11].

To go into more detail on plaque echogenicity, it has been noted that symptomatic lesions are typically associated with purely or predominantly hypoechoic plaques. There has been an effort to measure plaque echogenicity quantitatively. Biasi et al. [12] used longitudinal images of the plaque and vessel wall and measured the gray-scale median (GSM). The GSM of the blood pool was 0 to 5, and the GSM of the adventitia of the wall was 185 to 195. What they found was that the stroke risk during carotid stenting procedures is dependent on the GSM of the plaque. Plaque with GSM values of 25 or less showed a $7.1 \%$ stroke risk while plaque with GSM values more than 25 showed only a 1.5\% stroke risk. This means that echolucent plaque is more vulnerable [11] (Fig. 3B).

Recently, three-dimensional (3D) ultrasonography has been used for measuring plaque volume [13-15] (Fig. 4). On a 2D grayscale image, plaque size can be measured based on length and height, but the total volume of the plaque cannot be measured. 3D ultrasonography showed good intra- and interobserver reproducibility for measuring total plaque volume [15].

The plaque volume can be used as a monitoring tool for atherosclerosis treatment. The plaque volume is known to increase without treatment and decrease with statin therapy [16]. 3D ultrasonography is thought to be useful for the monitoring of plaque and could also be useful for the evaluation of new treatments [17].

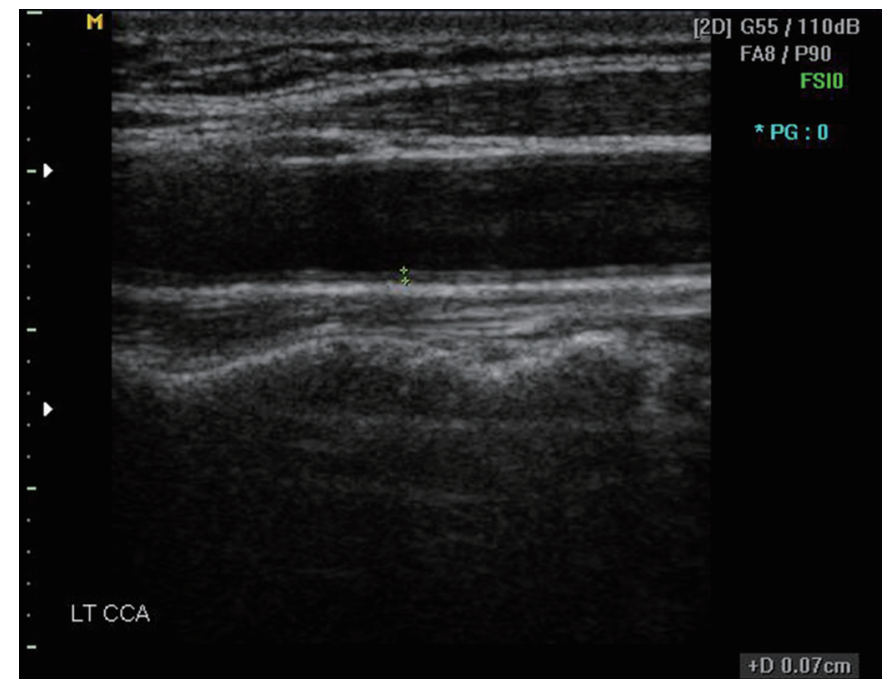

A

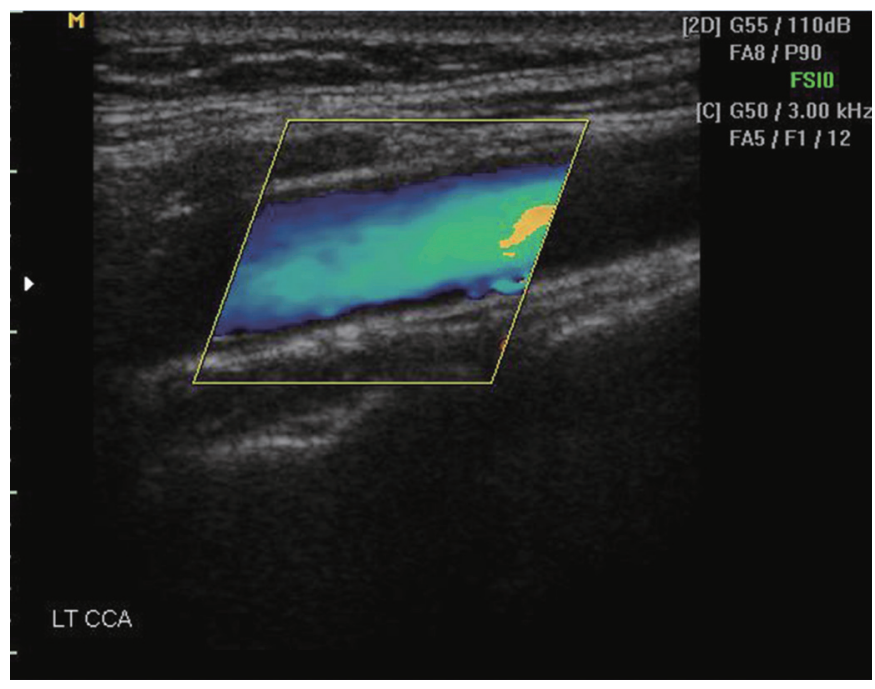

B

Fig. 5. Heel and toe technique. Tilting the probe from the head side to the toe side creates an angle between the probe surface and the vessel.

A. For intima-media thickness measurement, the probe surface should be parallel to the vessel. B. For detecting a color signal and measuring flow velocity, an angle of at least $30^{\circ}$ between the probe surface and the vessel is needed. 
3D ultrasonography volume measurements are more sensitive than IMT for the evaluation of carotid plaque progression posttreatment. More specifically, while there was a significant change in the 3D plaque volume during the follow-up period, there was no change in the IMT.

3D ultrasonography also can be used for plaque characterization. The limitation of 2D gray-scale evaluation of plaque is that single or even multiple images cannot represent the entire plaque volume. Heliopoulos et al. [18] tried to measure the echogenicity of the entire volume of plaque with 3D ultrasonography. In 110 symptomatic and 104 asymptomatic patients with carotid plaque disease, they assessed the mean gray value of the whole plaque and found a higher incidence of low echoic plaque in symptomatic patients than in asymptomatic patients, suggesting a higher risk of cerebral ischemia from the low echoic plaque.

\section{Color Doppler Ultrasonography and Pulsed Wave Doppler Ultrasonography}

Color Doppler is color-encoded velocity information on a gray-scale image. Color Doppler is a good tool for visualizing the blood flow in the vessel and finding stenotic segments.

To obtain a proper color Doppler image, an adequate acoustic angle is essential. With a linear probe, to generate a gray-scale image, the sonic beam needs to be perpendicular to the skin. However, to obtain proper velocity information from color Doppler ultrasonography, the Doppler angle should be between $30^{\circ}$ and $60^{\circ}$. The carotid artery is not a deeply located structure, and ensuring the proper angle of the Doppler probe surface relative to the common carotid artery is not easy. In contrast to measuring the IMT position, in which it is better for the vessel wall to be parallel to the probe surface, there should be an angle between the probe surface and vessel in color Doppler ultrasonography. One helpful technique for achieving this angle is the heel and toe technique. The heel and

Table 1. Doppler criteria for diagnosis of ICA stenosis

\begin{tabular}{|c|c|c|c|}
\hline $\begin{array}{c}\text { Diameter } \\
\text { stenosis (\%) }\end{array}$ & PSV $(\mathrm{cm} / \mathrm{sec})$ & $\mathrm{EDV}(\mathrm{cm} / \mathrm{sec})$ & $\begin{array}{c}\text { ICA/CCA PSV } \\
\text { ratio }\end{array}$ \\
\hline Normal & $<125$ & $<40$ & $<2.0$ \\
\hline$<50$ & $<125$ & $<40$ & $<2.0$ \\
\hline $50-69$ & $125-230$ & $40-100$ & $2.0-4.0$ \\
\hline$\geq 70$ & $>230$ & $>100$ & $>4.0$ \\
\hline $\begin{array}{l}\text { Near total } \\
\text { occlusion }\end{array}$ & Variable & Variable & Variable \\
\hline Total occlusion & Undetectable & Undetectable & Not applicable \\
\hline
\end{tabular}

toe technique is a way of steering the probes. In the usual position for carotid artery scanning without any pressure, the probe surface will be parallel to the common carotid artery in most patients. Just pushing the head side edge or foot side edge will create a bit of an angle between the probe surface and vessel, and the optimal Doppler angle can be achieved (Fig. 5).

Adjusting the velocity range is one of the important ways of controlling Doppler ultrasonography parameters. One of the purposes of color Doppler ultrasonography is finding any stenotic segment in the vessel. Because the flow volume through the vessel is constant, the velocity of the flow is fastest at the stenotic segment. If the upper limit of the color velocity scale is just below that of the flow velocity in the normal vessel, the increased flow velocity in the stenotic segment will be above the upper limit of the velocity scale and there will be an aliasing artifact. If there is a segment showing an aliasing artifact at the proper velocity scale setting, it means that the segment is stenotic. The usual normal velocity of the common carotid artery is $30-40 \mathrm{~cm} / \mathrm{sec}$ [19], but the velocity scale setting should be adjusted for each patient.

However, to measure the exact flow velocity, we cannot rely on color Doppler imaging; we need pulsed wave Doppler. In pulsed wave Doppler, a small sample volume in the center of the vessel or in the stenotic segment will be used to check the velocity of the segment. The peak velocity is used for detecting significant stenosis. Angle correction is essential to measure the true flow velocity, and the angle correction should be along the flow direction, not along the vessel wall. The flow direction is usually not different from the vessel direction, but in cases of eccentric atherosclerotic plaque, the flow direction and vessel direction can be different.

Many criteria have been used for detecting significant stenosis

Table 2. Carotid Doppler report

\begin{tabular}{lll}
\hline \multicolumn{1}{c}{ Variable } & \multicolumn{1}{c}{ Right } & \multicolumn{1}{c}{ Left } \\
\hline Gray-scale imaging \\
Intima-media thickness \\
Atheroma or other findings \\
Doppler measurement \\
CCA-PSV \\
ICA-PSV \\
ICA-EDV \\
ICA/CCA PSV ratio \\
ECA-PSV
\end{tabular}




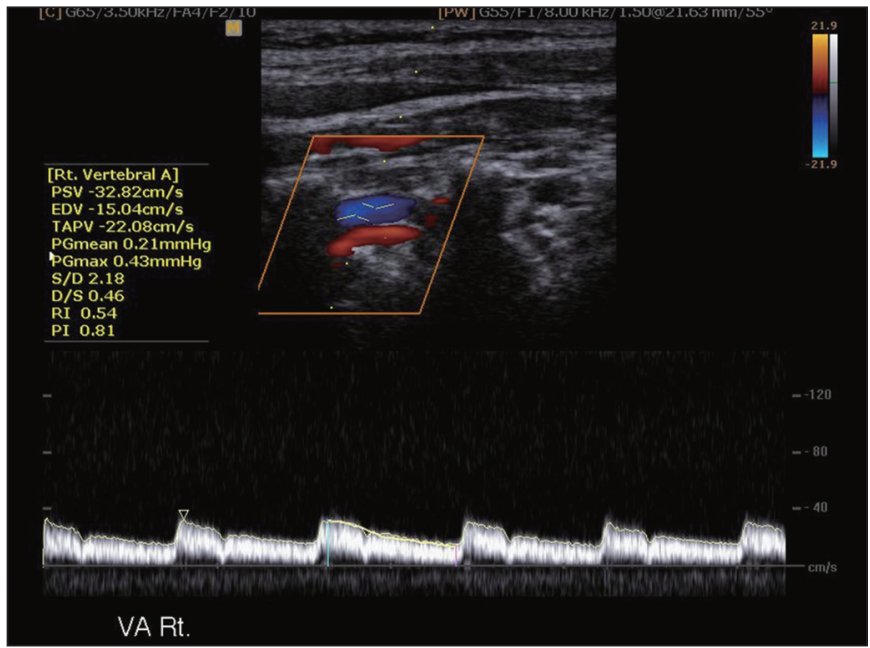

Fig. 6. Vertebral artery Doppler spectrum. The vertebral artery Doppler spectrum is similar to that of the internal carotid artery. It should be noted that the flow directions of the vertebral artery and vertebral vein are opposite each other.

of the ICA. In 2003, a consensus conference was held for Doppler ultrasonography diagnosis of carotid artery stenosis [20], and they proposed consensus velocity criteria for carotid artery stenosis, which has been used since then (Table 1). In these criteria, the peak systolic velocity is important. A peak systolic velocity of $125 \mathrm{~cm} /$ sec or higher in the ICA or twice as fast as that of the common carotid artery is thought to indicate possible significant stenosis of the ICA. A good example of a report form for carotid Doppler ultrasonography, as shown in Table 2, should include all the information on these criteria.

The vertebral artery Doppler spectrum has a low resistive pattern like that of the ICA (Fig. 6). In examining the vertebral artery, the flow direction is important because it can be reversed in case of subclavian steal syndrome [21].

\section{Pitfalls in Carotid Doppler Ultrasonography}

A calcified atheroma of the carotid artery can make it difficult to find a proper sonic window for color Doppler ultrasonography or pulsed wave Doppler. Trying different sonic windows such as posterolateral projection is helpful in some cases. If the obscured segment is less than $1 \mathrm{~cm}$ in size and there are normal waveforms at the proximal and distal parts of the plaque, it can be concluded that no significant stenosis exists. However, when the calcification is obscuring a large vascular segment, another imaging modality such as magnetic resonance angiography could be required.

In case of contralateral carotid artery stenosis, the PSV can be falsely elevated without significant stenosis. If there is critical stenosis of the ICA such as a residual luminal diameter of less than $0.7 \mathrm{~mm}$, the peak systolic velocity of the affected segment can show pseudo-normalization. Color Doppler ultrasonography could depict this critical stenosis of the ICA.

Carotid Doppler ultrasonography is a powerful modality for evaluating the carotid arteries. Carotid Doppler ultrasonography is useful not only for detecting carotid artery stenosis, but also for detecting atherosclerotic plaque including visualizing the intimamedia thickness as a biomarker for atherosclerosis. Radiologists should be familiar with the physics and clinical findings of Doppler ultrasonography in performing Doppler ultrasonography studies of the carotid arteries.

ORCID: Whal Lee: http://orcid.org/0000-0003-1285-5033

\section{Conflict of Interest}

No potential conflict of interest relevant to this article was reported.

\section{References}

1. Bots ML, Hoes AW, Koudstaal PJ, Hofman A, Grobbee DE. Common carotid intima-media thickness and risk of stroke and myocardial infarction: the Rotterdam Study. Circulation 1997;96:1432-1437.

2. Ross R. The pathogenesis of atherosclerosis: a perspective for the 1990s. Nature 1993;362:801-809.

3. Smith SC Jr, Greenland P, Grundy SM. AHA Conference Proceedings. Prevention conference $\mathrm{V}$. Beyond secondary prevention: identifying the high-risk patient for primary prevention: executive summary. American Heart Association. Circulation 2000;101:111-116.

4. Park AE, McCarthy WJ, Pearce WH, Matsumura JS, Yao JS. Carotid plaque morphology correlates with presenting symptomatology. J Vasc Surg 1998;27:872-878.

5. Gasecki AP, Eliasziw M, Barnett HJ. Risk factors for cervical atherosclerosis in patients with transient ischemic attack or minor ischemic stroke. Stroke 1994;25:226.

6. Eliasziw M, Streifler JY, Fox AJ, Hachinski VC, Ferguson GG, Barnett $\mathrm{HJ}$. Significance of plaque ulceration in symptomatic patients with high-grade carotid stenosis. North American Symptomatic Carotid Endarterectomy Trial. Stroke 1994;25:304-308.

7. O'Leary DH, Holen J, Ricotta JJ, Roe S, Schenk EA. Carotid bifurcation disease: prediction of ulceration with B-mode US. Radiology 1987;162:523-525.

8. O'Donnell TF Jr, Erdoes L, Mackey WC, McCullough J, Shepard A, Heggerick P, et al. Correlation of B-mode ultrasound imaging and arteriography with pathologic findings at carotid endarterectomy. Arch Surg 1985;120:443-449.

9. Comerota AJ, Katz ML, White JV, Grosh JD. The preoperative diagnosis of the ulcerated carotid atheroma. J Vasc Surg 
1990;11:505-510.

10. Widder B, Paulat K, Hackspacher J, Hamann H, Hutschenreiter S, Kreutzer $C$, et al. Morphological characterization of carotid artery stenoses by ultrasound duplex scanning. Ultrasound Med Biol 1990;16:349-354.

11. Gray-Weale AC, Graham JC, Burnett JR, Byrne K, Lusby RJ. Carotid artery atheroma: comparison of preoperative B-mode ultrasound appearance with carotid endarterectomy specimen pathology. J Cardiovasc Surg (Torino) 1988;29:676-681.

12. Biasi GM, Froio A, Diethrich EB, Deleo G, Galimberti S, Mingazzini $P$, et al. Carotid plaque echolucency increases the risk of stroke in carotid stenting: the Imaging in Carotid Angioplasty and Risk of Stroke (ICAROS) study. Circulation 2004;110:756-762.

13. Delcker A, Diener HC. Quantification of atherosclerotic plaques in carotid arteries by three-dimensional ultrasound. $\mathrm{Br} J$ Radiol 1994;67:672-678.

14. Griewing B, Schminke U, Morgenstern C, Walker ML, Kessler C. Three-dimensional ultrasound angiography (power mode) for the quantification of carotid artery atherosclerosis. J Neuroimaging 1997;7:40-45.

15. Landry A, Spence JD, Fenster A. Measurement of carotid plaque volume by 3-dimensional ultrasound. Stroke 2004;35:864-869.

16. Pollex RL, Spence JD, House AA, Fenster A, Hanley AJ, Zinman B, et al. A comparison of ultrasound measurements to assess carotid atherosclerosis development in subjects with and without type 2 diabetes. Cardiovasc Ultrasound 2005;3:15.

17. Makris GC, Lavida A, Griffin M, Geroulakos G, Nicolaides AN. Threedimensional ultrasound imaging for the evaluation of carotid atherosclerosis. Atherosclerosis 2011;219:377-383.

18. Heliopoulos J, Vadikolias K, Mitsias P, Artemis D, Tripsianis G, Piperidou $C$, et al. A three-dimensional ultrasonographic quantitative analysis of non-ulcerated carotid plaque morphology in symptomatic and asymptomatic carotid stenosis. Atherosclerosis 2008;198:129-135.

19. Kim S, Lee S, Choi HS, Jung SL, Ahn KJ, Kim BS. Pseudostenosis at the origin of the vertebral artery on contrast-enhanced mra: correlation with aortic motion on dynamic 3D time-resolved contrast-enhanced MRA. J Korean Soc Magn Reson Med 2012;16: 236-242.

20. Grant EG, Benson CB, Moneta GL, Alexandrov AV, Baker JD, Bluth El, et al. Carotid artery stenosis: gray-scale and Doppler US diagnosis--Society of Radiologists in Ultrasound Consensus Conference. Radiology 2003;229:340-346.

21. Jeon SW, Chang HW, Kim MJ, Cho J. Persistent proatlantal artery in magnetic resonance angiography: a case report. J Korean Soc Magn Reson Med 2013;17:55-58. 